Novi SAD J. MATH.

VoL. 47, No. 1, 2017, 177-188

\title{
GENERALIZED SCHWARZ INEQUALITIES FOR GENERALIZED SEMI-INNER PRODUCTS ON GROUPOIDS CAN BE DERIVED FROM AN EQUALITY'
}

\author{
Zoltán Boros $\$^{2}$ and Árpád Száz沎
}

\begin{abstract}
By introducing an appropriate notion of generalized semiinner products on groupoids, we shall prove a simple equality which can be used to easily derive some generalized forms of the famous Schwarz inequality.

In the case of groups, these generalized forms will turn out to be sufficient to prove the subadditivity of the induced generalized seminorms. Thus, some results on inner product spaces can be extended to inner product groups.

AMS Mathematics Subject Classification (2010): 20L05; 46C50; 39B32; 39B62
\end{abstract}

Key words and phrases: groupoids; generalized inner products; Schwarz inequalities; seminorms

\section{Introduction}

Semi-inner products on groups were first introduced by the second author in 18 to prove a natural generalization of a basic theorem of Maksa and Volkmann [16] on additive functions without any particular tricks preferred by functional equationalists.

In 18 , the second author claimed that even a weakened form of Schwarz inequality cannot be proved for semi-inner products on groups. Moreover, he asked several mathematicians in Debrecen and Cluj-Napoca, at a conference, to justify his statement by providing an appropriate example.

However, the first author could disprove this claim by justifying a weak form of Schwarz inequality [3] which is slightly more than that is needed to prove the subadditivity of the induced generalized seminorm. Thus, some results on inner product spaces can be extended to inner product groups.

This weak Schwarz inequality, which was first presented in our joint report [6], has later been substantially generalized by the second author by taking

\footnotetext{
${ }^{1}$ The work of the authors has been supported by the Hungarian Scientific Research Fund (OTKA) Grant K-111651.

${ }^{2}$ Department of Mathematics, University of Debrecen, H-4002 Debrecen, Pf. 400, Hungary e-mail: zboros@science.unideb.hu

${ }^{3}$ Department of Mathematics, University of Debrecen, H-4002 Debrecen, Pf. 400, Hungary e-mail: szaz@science.unideb.hu

${ }^{4}$ Corresponding author
} 
groupoids instead of groups, and using the smallest denominator function of [4, 5]. The corresponding results were presented in our joint reports [7] and 8

Moreover, the second author has also noticed that instead of the generalized Schwarz inequalities it is more convenient to prove first an equality which easily yields the corresponding inequalities [19]. Therefore, our technical report [8] has to be completely rewritten.

For this, in the present paper, we shall suppose that $X$ is an additively written groupoid and $P$ is a function of $X^{2}$ to $\mathbb{C}$ such that, under the notations

$$
\Delta_{P}(x)=P(x, x) \quad \text { and } \quad P_{1}(x, y)=2^{-1}(P(x, y)+\overline{P(x, y)}),
$$

for any $n \in \mathbb{N}$ and $x, y \in X$ we have

(a) $\Delta_{P}(x)=\Delta_{P_{1}}(x)$,

(b) $P_{1}(n x, y)=n P_{1}(x, y)=P_{1}(x, n y)$,

(c) $\Delta_{P}(x+y)=\Delta_{P}(x)+\Delta_{P}(y)+2 P_{1}(x, y)$.

By using this generalized semi-inner product $P$ and the notations

$$
\alpha_{P}(x, y)=\inf _{r \in \mathbb{Q}_{+}}\left(r \Delta_{P}(x)+r^{-1} \Delta_{P}(y)\right)
$$

and

$$
\beta_{P}(x, y)=\inf _{r \in \mathbb{Q}_{+}} \frac{1}{m(r) n(r)} \Delta_{P}(m(r) x+n(r) y),
$$

with

$$
n(r)=\min \{n \in \mathbb{N}: \quad r n \in \mathbb{N}\} \quad \text { and } \quad m(r)=r n(r),
$$

we shall show that

$$
\beta_{P}(x, y)=\alpha_{P}(x, y)+2 P_{1}(x, y)
$$

for all $x, y \in X$.

Hence, by assuming that $\Delta_{P}$ is nonnegative and proving that

$$
\alpha_{P}(x, y)=2 \sqrt{\Delta_{P}(x) \Delta_{P}(y)},
$$

for all $x, y \in X$, we can already derive that

$$
-P_{1}(x, y) \leq p(x) p(y)
$$

for all $x, y \in X$ with $p(x)=\sqrt{\Delta_{P}(x)}$.

Thus, if in addition $P_{1}$ is biodd (or more generally $\Delta_{P}$ is even and $P_{1}$ is odd in its fist argument), then we can also state that

$$
\left|P_{1}(x, y)\right| \leq p(x) p(y)
$$

for all $x, y \in X$. In [7, Example 5.6], the first author has shown that the function $P_{2}$ need not have similar properties even if, in particular, $P$ is an $\mathbb{R}$-bihomogeneous semi-inner product on $\mathbb{R}^{2}$ with nonnegative diagonalization. 


\section{A generalized semi-inner product}

Our subsequent generalization of the ordinary semi-inner products [17, 6, 20. 7. may be modified according to the ideas of Lumer [15, Giles [12], Dragomir [9], Bognár [2], Antoine and Grossmann [1] and Drygas [10].

Notation 2.1. Let $X$ be an additively written groupoid. Moreover, suppose that $P$ is a function of $X^{2}$ to $\mathbb{C}$.

For any $n \in \mathbb{N}$ and $x \in X$, define

$$
n x=x \quad \text { if } \quad n=1 \quad \text { and } \quad n x=(n-1) x+x \quad \text { if } \quad n>1 .
$$

Moreover, for any $x, y \in X$, define

$$
\Delta_{P}(x)=P(x, x) \quad \text { and } \quad P_{1}(x, y)=2^{-1}(P(x, y)+\overline{P(x, y)}) .
$$

Furthermore, suppose that, for any $n \in \mathbb{N}$ and $x, y \in X$, we have

(a) $\Delta_{P}(x)=\Delta_{P_{1}}(x)$,

(b) $P_{1}(n x, y)=n P_{1}(x, y)=P_{1}(x, n y)$,

(c) $\Delta_{P}(x+y)=\Delta_{P}(x)+\Delta_{P}(y)+2 P_{1}(x, y)$.

Remark 2.2. Properties (a), (b) and (c) will be called the the reality of $\Delta_{P}$, $\mathbb{N}$-bihomogeneity of $P_{1}$ and polarization identity for $P$, respectively.

The functions $\Delta_{P}$ and $P_{1}$ will be called the diagonalization and real part (first coordinate function) of $P$, respectively. Moreover, the function $P$ itself will be called a generalized semi-inner product on $X$.

If, in particular, the groupoid $X$ has a zero element 0 , then the generalized semi-inner product $P$ will be called a generalized inner product if $\Delta_{P}(x)=0$ implies $x=0$ for all $x \in X$.

Some further useful additional properties of $X$ and $P$ will be assumed gradually as the forthcoming definitions and theorems need. For instance, we may naturally assume that $X$ is a group and $P_{1}$ is $\mathbb{Z}$-bihomogeneous.

The following theorem will clarify the appropriateness of our former assumptions on $P$.

Theorem 2.3. If $Q$ is a semi inner product on $X$ in the sense that $Q$ is a conjugate-symmetric function of $X^{2}$ to $\mathbb{C}$ such that $Q$ is additive in its first argument, then $Q$ is a generalized semi-inner product on $X$ with several useful additional properties.

Proof. By the conjugate-symmetry of $Q$, for any $x, y \in X$, we have

$$
Q(y, x)=\overline{Q(x, y)} .
$$

Hence, in particular, it clear that

$$
Q(x, x)=\overline{Q(x, x)}, \quad \text { and thus } \quad Q(x, x)=Q_{1}(x, x) .
$$


Therefore, property (a) holds for $Q$.

Moreover, we can also at once see that

$$
Q_{1}(x, y)=2^{-1}(Q(x, y)+\overline{Q(x, y)})=2^{-1}(Q(x, y)+Q(y, x))
$$

Thus, $Q_{1}$ is just the symmetric part of $Q$. Concerning the imaginary part (second coordinate function) $Q_{2}$ of $Q$, we can quite similarly see that

$$
Q_{2}(x, y)=(i 2)^{-1}(Q(x, y)-\overline{Q(x, y)})=i^{-1} 2^{-1}(Q(x, y)-Q(y, x)) .
$$

for all $x, y \in X$. Therefore, $i Q_{2}$ is just the skew-symmetric part of $Q$ [21, p. 294].

Furthermore, by using the conjugate-symmetry and the additivity of $Q$ in its first argument, we can easily see that

$$
\begin{aligned}
Q(x, y+z)=\overline{Q(y+z, x)} & =\overline{Q(y, x)+Q(z, x)}= \\
& =\overline{Q(y, x)}+\overline{Q(z, x)}=Q(x, y)+Q(x, z)
\end{aligned}
$$

for all $x, y, z \in X$. Therefore, $Q$ is additive in its second argument too. That is, $Q$ is actually biadditive.

Thus, for any $x, y \in X$, the first and second partial functions of $Q$, defined by

$$
\varphi_{Q}(u)=Q(u, y) \quad \text { and } \quad \psi_{Q}(v)=Q(x, v)
$$

for all $u, v \in X$, are additive functions of $X$ to $\mathbb{C}$.

Hence, since an additive function of one groupoid to another can easily be seen to be $\mathbb{N}$-homogeneous, it is clear that $\varphi_{Q}$ and $\psi_{Q}$ are $\mathbb{N}$-homogeneous. Therefore, $Q$ and thus its coordinate functions $Q_{1}$ and $Q_{2}$ are, as well, $\mathbb{N}$ bihomogeneous. Thus, in particular, property (b) also holds.

Moreover, by using the biadditivity of $Q$ and our former observation on $Q_{1}$, we can also see that

$$
\begin{aligned}
& \Delta_{Q}(x+y)=Q(x+y, x+y)=Q(x, x+y)+Q(y, x+y) \\
= & Q(x, x)+Q(x, y)+Q(y, x)+Q(y, y)=\Delta_{Q}(x)+2 Q_{1}(x, y)+\Delta_{Q}(y)
\end{aligned}
$$

for all $x, y \in X$. Thus, property (c) also holds for $Q$.

Remark 2.4. Note that, in particular, if $X$ is a group, then instead of the $\mathbb{N}$-bihomogeneity of $Q$ we can also state the $\mathbb{Z}$-bihomogeneity of $Q$.

Therefore, in particular, if $X$ is a group, then instead of property (b) it is convenient to assume the $\mathbb{Z}$-bihomogenity of $P_{1}$.

Moreover, if $P$ is not conjugate symmetric, then in Notation 2.1 it may be convenient to take the symmetric part of $P$ instead of $P_{1}$.

The appropriateness of assumptions (a)-(c) is also apparent from the following 
Theorem 2.5. For any $n, m \in \mathbb{N}$ and $x, y \in X$,

(1) $\Delta_{P}(n x)=n^{2} \Delta_{P}(x)$,

(2) $\Delta_{P}(n(x+y))=\Delta_{P}(n x+n y)$,

(3) $\Delta_{P}(m x+n y)=m^{2} \Delta_{P}(x)+n^{2} \Delta_{P}(y)+2 m n P_{1}(x, y)$.

Proof. By (a) and (b), we have

$$
\begin{aligned}
\Delta_{P}(n x)=\Delta_{P_{1}}(n x)=P_{1}(n x & , n x)=n P_{1}(x, n x) \\
& =n^{2} P_{1}(x, x)=n^{2} \Delta_{P_{1}}(x)=n^{2} \Delta_{P}(x),
\end{aligned}
$$

and thus (1) is true.

To prove (2) and (3), note that by (1) and (c) we have

$$
\Delta_{P}(n(x+y))=n^{2} \Delta_{P}(x+y)=n^{2} \Delta_{P}(x)+n^{2} \Delta_{P}(y)+2 n^{2} P_{1}(x, y) .
$$

Moreover, by (c), (1) and (b), we also have

$$
\begin{aligned}
\Delta_{P}(m x+n y)=\Delta_{P}(m x)+ & \Delta_{P}(n y)+2 P_{1}(m x, n y) \\
& =m^{2} \Delta_{P}(x)+n^{2} \Delta_{P}(y)+2 m n P_{1}(x, y),
\end{aligned}
$$

and thus, in particular,

$$
\Delta_{P}(n x+n y)=n^{2} \Delta_{P}(x)+n^{2} \Delta_{P}(y)+2 n^{2} P_{1}(x, y) .
$$

Remark 2.6. If, in particular, $P_{1}$ is symmetric, then because of (3) we can also state that

$$
\Delta_{P}(n y+m x)=\Delta_{P}(m x+n y) .
$$

Remark 2.7. While if, in particular, $X$ is a group and $P_{1}$ is $\mathbb{Z}$-bihomogeneous, then equalities (2) and (3) can also be stated for all $n, m \in \mathbb{Z}$.

\section{A general equality leading to Schwarz inequalities}

The appropriateness of the following definition will become quite obvious from the proof of the forthcoming Theorem 3.4

Definition 3.1. For any $x, y \in X$, we define

$$
\alpha_{P}(x, y)=\inf _{r \in \mathbb{Q}_{+}}\left(r \Delta_{P}(x)+r^{-1} \Delta_{P}(y)\right)
$$

and

$$
\beta_{P}(x, y)=\inf _{r \in \mathbb{Q}_{+}} \frac{1}{m(r) n(r)} \Delta_{P}(m(r) x+n(r) y)
$$

with

$$
n(r)=\min \{n \in \mathbb{N}: \quad r n \in \mathbb{N}\} \quad \text { and } \quad m(r)=r n(r) .
$$


Remark 3.2. The natural numbers $n(r)$ and $m(r)$ are called the smallest denominator and the associated numerator of $r \in \mathbb{Q}_{+}$, respectively.

Several remarkable properties and important applications of their obvious extensions to $r \in \mathbb{Q}$ have been established in our former papers [4] and [5] .

For instance, we have proved that if $r=k / l$, for some $k \in \mathbb{Z}$ and $l \in \mathbb{N}$, then

$$
m(r)=k /(k ; l) \quad \text { and } \quad n(r)=l /(k ; l),
$$

where $(k ; l)$ is the greatest common divisor of $k$ and $l$. Thus, in particular $m(r)$ and $n(r)$ are relatively prime in the sense that $(m(r) ; n(r))=1$.

Remark 3.3. In the next section, we shall see that the extended real number $\alpha_{P}(x, y)$ can be quite easily determined.

However, concerning $\beta_{P}(x, y)$, we can only note that if $\Delta_{P}(m x+n y) \geq$ 0 for all $m, n \in \mathbb{N}$ with $(m ; n)=1$, then $\beta_{P}(x, y) \geq 0$.

Fortunately, this simple observation will already allow us to establish some applicable consequences of the following

Theorem 3.4. For any $x, y \in X$, we have

$$
\beta_{P}(x, y)=\alpha_{P}(x, y)+2 P_{1}(x, y) .
$$

Proof. By (3) in Theorem 2.5, for any $r \in \mathbb{Q}_{+}$, we have

$\Delta_{P}(m(r) x+n(r) y)=m(r)^{2} \Delta_{P}(x)+n(r)^{2} \Delta_{P}(y)+2 m(r) n(r) P_{1}(x, y)$, and thus

$$
\begin{aligned}
\frac{1}{m(r) n(r)} \Delta_{P}(m(r) x+n(r) y)= & \frac{m(r)}{n(r)} \Delta_{P}(x)+\frac{n(r)}{m(r)} \Delta_{P}(y)+2 P_{1}(x, y) \\
& =r \Delta_{P}(x)+r^{-1} \Delta_{P}(y)+2 P_{1}(x, y) .
\end{aligned}
$$

Hence, by using a theorem on infimum, we can already infer that

$$
\begin{gathered}
\beta_{P}(x, y)=\inf _{r \in \mathbb{Q}_{+}} \frac{1}{m(r) n(r)} \Delta_{P}(m(r) x+n(r) y) \\
=\inf _{r \in \mathbb{Q}_{+}}\left(r \Delta_{P}(x)+r^{-1} \Delta_{P}(y)+2 P_{1}(x, y)\right) \\
=\inf _{r \in \mathbb{Q}_{+}}\left(r \Delta_{P}(x)+r^{-1} \Delta_{P}(y)\right)+2 P_{1}(x, y)=\alpha_{P}(x, y)+2 P_{1}(x, y) .
\end{gathered}
$$

From this theorem, we can immediately derive

Corollary 3.5. If $x, y \in X$ such that either $\alpha_{P}(x, y)$ or $\beta_{P}(x, y)$ is finite, then

$$
-P_{1}(x, y)=2^{-1}\left(\alpha_{P}(x, y)-\beta_{P}(x, y)\right) .
$$


Hence, by Remark 3.3 , it is clear that, in particular, we also have

Corollary 3.6. If $x, y \in X$ such that $\Delta_{P}(m x+n y) \geq 0$ for all $m, n \in \mathbb{N}$ with $(m ; n)=1$, then

$$
-P_{1}(x, y) \leq 2^{-1} \alpha_{P}(x, y) .
$$

Now, by using this corollary, we can also easily prove the following

Theorem 3.7. If $X$ is a group, $\Delta_{P}$ is nonnegative and $P_{1}$ is biodd, then for any $x, y \in X$ we have

$$
\left|P_{1}(x, y)\right| \leq 2^{-1} \alpha_{P}(x, y) .
$$

Proof. By property (a) and the biodness of $P_{1}$, we have

$$
\Delta_{P}(-x)=\Delta_{P_{1}}(-x)=P_{1}(-x,-x)=P_{1}(x, x)=\Delta_{P_{1}}(x)=\Delta_{P}(x)
$$

for all $x \in X$. Therefore, $\Delta_{P}$ is even, and thus $\alpha_{P}$ is bieven.

Moreover, by the nonnegativity of $\Delta_{P}$ and Corollary 3.6, we have

$$
-P_{1}(x, y) \leq 2^{-1} \alpha_{P}(x, y)
$$

for all $x, y \in X$.

Hence, by using the oddness of $P_{1}$ and the evenness of $\alpha_{P}$ in their first arguments, we can already see that

$$
P_{1}(x, y)=-P_{1}(-x, y) \leq 2^{-1} \alpha_{P}(-x, y)=2^{-1} \alpha_{P}(x, y)
$$

also holds for all $x, y \in X$. Therefore, by the definition of the absolute value, the required inequality is also true.

Remark 3.8. Now, to obtain some applicable consequences of Theorem 3.7, we need only to compute $\alpha_{P}(x, y)$ for all $x, y \in X$.

\section{The determination of $\alpha_{P}(x, y)$}

To compute the values of $\alpha_{P}$, it seems convenient to prove first the following

Lemma 4.1. For any $x, y \in X$, we have

$$
\alpha_{P}(x, y)=\inf _{\lambda>0}\left(\lambda \Delta_{P}(x)+\lambda^{-1} \Delta_{P}(y)\right) .
$$

Proof. Because of the lower bound property of infimum, we have

$$
\alpha_{P}(x, y) \leq r \Delta_{P}(x)+r^{-1} \Delta_{P}(y)
$$

for all $r \in \mathbb{Q}_{+}$. Hence, by using the sequential denseness of $\mathbb{Q}$ in $\mathbb{R}$ and the sequential continuity of the operations in $\mathbb{R}$, we can infer that

$$
\alpha_{P}(x, y) \leq \lambda \Delta_{P}(x)+\lambda^{-1} \Delta_{P}(y)
$$


also holds for all $\lambda \in \mathbb{R}_{+}$. Therefore, by the maximality property of infimum, we have

$$
\alpha_{P}(x, y) \leq \inf _{\lambda \in \mathbb{R}_{+}}\left(\lambda \Delta_{P}(x)+\lambda^{-1} \Delta_{P}(y)\right) .
$$

Moreover, by using the inclusion $\mathbb{Q}_{+} \subseteq \mathbb{R}_{+}$and the definition of infimum, we can also easily see that the converse inequality also holds. Therefore, the required equality is also true.

Remark 4.2. Concerning $\beta_{P}$, we can also prove that

$$
\beta_{P}(x, y)=\inf _{m, n \in \mathbb{N}} \frac{1}{m n} \Delta_{P}(m x+n y) .
$$

However, because of Theorem 3.4 this fact seems to be of no importance.

Now, by using Lemma 4.1 and some basic facts from calculus, we can also easily prove the following

Theorem 4.3. For any $x, y \in X$, we have

(1) $\alpha_{P}(x, y)=-\infty$ if either $\Delta_{P}(y)<0$ or $\Delta_{P}(x)<0$,

(2) $\alpha_{P}(x, y)=2 \sqrt{\Delta_{P}(x) \Delta_{P}(y)}$ if $\Delta_{P}(x) \geq 0$ and $\Delta_{P}(y) \geq 0$.

Proof. Here, we shall prove somewhat more than what is stated. For this, define

$$
a=\Delta_{P}(x), \quad b=\Delta_{P}(y) \quad \text { and } \quad c=\alpha_{P}(x, y) .
$$

Moreover, define

$$
f(\lambda)=a \lambda+b \lambda^{-1}
$$

for all $\lambda>0$.

Then, by Lemma 4.1 , we have

$$
c=\inf _{\lambda>0} f(\lambda) .
$$

Moreover, we can note that $f$ is a differentiable function of $\mathbb{R}_{+}$such that

$$
f^{\prime}(\lambda)=a-b \lambda^{-2}
$$

for all $\lambda>0$. Therefore,

$$
f^{\prime}(\lambda)<0 \Longleftrightarrow a \lambda^{2}<b \quad \text { and } \quad f^{\prime}(\lambda)>0 \Longleftrightarrow b<a \lambda^{2} .
$$

Hence, if $a>0$ and $b>0$, then by defining $\lambda_{0}=\sqrt{b / a}$ we can see that $f$ is strictly decreasing on $\left.]-\infty, \lambda_{0}\right]$ and $f$ is strictly increasing on $\left[\lambda_{0},+\infty[\right.$. Therefore,

$$
f\left(\lambda_{0}\right)=a \lambda_{0}+b \lambda_{0}^{-1}=2 \sqrt{a b}
$$

is a strict global minimum of $f$. Thus, in particular, $c=2 \sqrt{a b}$.

Moreover, if $a=0$ and $b>0$, then we can see that $f$ is strictly decreasing on $\mathbb{R}_{+}$and $\lim _{\lambda \rightarrow+\infty} f(\lambda)=0$. Thus, in particular, $c=0=2 \sqrt{a b}$. 
While, if $a>0$ and $b=0$, then $f$ is strictly increasing on $\mathbb{R}_{+}$and $\lim _{\lambda \rightarrow 0} f(\lambda)=0$. Thus, in particular, $c=0=2 \sqrt{a b}$.

On the other hand, if either $a<0$ or $b<0$, then by not establishing the monotonicity properties $f$, we only note that $c=-\infty$. Namely, we evidently have

$$
\lim _{\lambda \rightarrow 0} f(\lambda)=-\infty \quad \text { if } \quad b<0, \quad \text { and } \quad \lim _{\lambda \rightarrow+\infty} f(\lambda)=-\infty \quad \text { if } \quad a<0 .
$$

Thus, summarizing the above observations, we can state that

$c=2 \sqrt{a b} \quad$ if $\quad a \geq 0$ and $b \geq 0, \quad$ and $\quad c=-\infty$ if either $a<0$ or $b<0$.

Therefore, the required assertions are also true.

Remark 4.4. Now, as an immediate consequence of Theorems 3.4 and 4.3 , we can also state that

(1) $\beta_{P}(x, y)=-\infty$ if either $\Delta_{P}(y)<0$ or $\Delta_{P}(x)<0$,

(2) $\beta_{P}(x, y)=2 \sqrt{\Delta_{P}(x) \Delta_{P}(y)}+2 P_{1}(x, y)$ if $\Delta_{P}(x) \geq 0$ and $\Delta_{P}(y) \geq 0$.

\section{The induced generalized seminorm}

By Theorem 4.3, we may naturally introduce the following

Definition 5.1. If, in particular, $\Delta_{P}$ is nonnegative, then for any $x \in X$ we define

$$
p(x)=\sqrt{\Delta_{P}(x)} .
$$

Remark 5.2. The function $p$ will be called the generalized seminorm derived from $P$.

Thus, as an immediate consequence of Corollary 3.6 and and Theorem 4.3 . we can state

Theorem 5.3. If $\Delta_{P}$ is nonnegative, then for any $x, y \in X$ we have

$$
-P_{1}(x, y) \leq p(x) p(y)
$$

Moreover, by Theorems 3.7 and 4.3 , we can also state

Theorem 5.4. If $X$ is a group, $\Delta_{P}$ is nonnegative and $P_{1}$ biodd, then for any $x, y \in X$ we have

$$
\left|P_{1}(x, y)\right| \leq p(x) p(y)
$$

Furthermore, by using Theorem 2.5, we can also easily establish 
Theorem 5.5. If $\Delta_{P}$ is nonnegative, then for any $n, m \in \mathbb{N}$ and $x, y \in X$ we have

(1) $p(n x)=n p(x)$,

(2) $p(n(x+y))=p(n x+n y)$,

(3) $p(m x+n y)^{2}=m^{2} p(x)^{2}+n^{2} p(y)^{2}+2 m n P_{1}(x, y)$.

Remark 5.6. If, in particular, $P_{1}$ is symmetric, then because of (3) we can also state that

$$
p(n y+m x)=p(m x+n y) .
$$

Remark 5.7. While if, in particular, $X$ is a group and $P_{1}$ is $\mathbb{Z}$-bihomogeneous, then instead of (1) we can also state that

$$
p(n x)=|n| p(x)
$$

for all $n \in \mathbb{Z}$. Moreover, equalities (2) and (3) remain valid for all $n, m \in \mathbb{Z}$.

Now, by using Theorems 5.4 and 5.5, we can also prove the following

Theorem 5.8. If $X$ is a group, $\Delta_{P}$ is nonnegative and $P_{1}$ is biodd, then for any $x, y \in X$ we have
(1) $p(x+y) \leq p(x)+p(y)$,
(2) $|p(x)-p(y)| \leq p(x-y)$.

Proof. By Theorems 5.5 and 5.4 it is clear that

$$
\begin{aligned}
p(x+y)^{2}=p(x)^{2}+ & p(y)^{2}+2 P_{1}(x, y) \\
& \leq p(x)^{2}+p(y)^{2}+2 p(x) p(y)=(p(x)+p(y))^{2} .
\end{aligned}
$$

Therefore, by the nonnegativity of $p$, inequality (1) is also true.

Now, by using (1), we can also easily see that

$p(x)=p(x-y+y) \leq p(x-y)+p(y), \quad$ and thus $\quad p(x)-p(y) \leq p(x-y)$.

Hence, it is clear that

$$
-(p(x)-p(y))=p(y)-p(x) \leq p(y-x)=p(-(x-y))=p(x-y),
$$

and thus (2) is also true. Namely, by the bioddness of $P_{1}$, the function $\Delta_{P}$ is even, and thus $p$ is also even.

Remark 5.9. Note that in Theorem 3.7] and thus also in Theorems 5.4 and 5.8, instead of the bioddness of $P_{1}$, it is enough to assume only that $\Delta_{P}$ is even and $P_{1}$ is odd in its first argument.

Note. Meantime, we observed that, besides Boros [3] and Ger [1], Kurepa [13, 14, also proved Schwarz inequalities on groups.

The results of Kurepa strongly suggest that instead of semi-inner products, it may be more convenient to start with quadratic functions. 


\section{References}

[1] Antoine, J.-P., Grossmann, A., Partial inner product spaces. I. General properties. J. Funct. Anal. 23 (1976), 369-378.

[2] Bognár, J, Indefinite Inner Product Spaces. Berlin: Springer, 1974.

[3] Boros, Z., Schwarz inequality over groups. Talk held at the Conference on Inequalities and Applications, dedicated to the 75th birthday of L.Losonczi and the 60th birthday of Zs. Páles, Hajdúszoboszló, Hungary, 2016.

[4] Boros, Z., Száz, Á., The smallest denominator function and the Riemann function. Acta Math. Acad. Paedagog. Nyházi. (N.S.) 14 (1998), 1-7.

[5] Boros, Z., Száz, Á., Some number theoretic applications of the smallest denominator function. Acta Math. Acad. Paedagog. Nyházi. (N.S.) 15 (1999), 19-267.

[6] Boros, Z., Száz, Á., Semi-inner products and their induced seminorms and semimetrics on groups. Tech. Rep., Inst. Math., Univ. Debrecen 2016/6, 11 pp.

[7] Boros, Z., Száz, Á., A weak Schwarz inequality for semi-inner products on groupoids. Tech. Rep., Inst. Math., Univ. Debrecen 2016/7, 10 pp.

[8] Boros, Z., Száz, Á., A most general Schwarz inequality for semi-inner products on groupoids. Tech. Rep., Inst. Math., Univ. Debrecen 2016/8, 9 pp.

[9] Dragomir, S. S., Semi-Inner Products and Applications. Hauppauge, NY: Nova Science Publishers, 2004.

[10] Drygas, H., Quasi-inner products and their applications. In: Advances in Multivariate Statistical Analysis, Theory Decis. Lib. Ser. B, Math. Statis. Methods. (A. K. Gupta, ed.), pp. 13-30. Dorrecht: Reidel, 1987.

[11] Ger, R., On a problem of Navid Safaei. Talk held at the Conference on Inequalities and Applications, dedicated to the 75th birthday of L. Losonczi and the 60th birthday of Zs. Páles, Hajdúszoboszló, Hungary, 2016.

[12] Giles, J.R., Classes of semi-inner-product spaces. Trans. Amer. Math, Soc. 129 (1967), 436-446.

[13] Kurepa, S., Quadratic and sesquilinear functionals. Glasnik Mat. 20 (1965), 79-91.

[14] Kurepa, S., On bimorphisms and quadratic forms on groups. Aequationes Math. 9 (1973), 30-45.

[15] Lumer, G., Semi-inner-product spaces. Trans. Amer. Math, Soc. 100 (1961), $29-43$.

[16] Maksa, Gy., Volkmann, P., Characterizations of group homomorphisms having values in an inner product space. Publ. Math. Debrecen 56 (2000), 197-200.

[17] Száz, Á., An instructive treatment of convergence, closure and orthogonality in semi-inner product spaces. Tech. Rep., Inst. Math., Univ. Debrecen, 2006/2, 29 pp.

[18] Száz, Á., Generalization of a theorem of Maksa and Volkmann on additive functions. Tech. Rep., Inst. Math., Univ. Debrecen 2016/5, 6 pp. (An improved and enlarged version is available from the author.)

[19] Száz, Á., Schwarz inequality has to be replaced by an equality. Lecture at the Seminar on Analysis, Debrecen, 2016. 
[20] Száz, Á., Remarks and problems at the Conference on Inequalities and Applications, Hajdúszoboszló, Hungary 2016. Tech. Rep., Inst. Math., Univ. Debrecen, 2016/9, 34 pp.

[21] Stetkaer, H., Functional Equations on Groups. New Jersey: World Scientific 2013.

Received by the editors January 5, 2017

First published online January 24, 2017 